\title{
INVITED ABSTRACTS
}

of reports reveals that structured reporting has undeniable advantages over free-text reporting. But, why this method has not been widely adopted?

Objectives: By listening to this lecture, the audience is expected to:

1. List the barriers to using structured reporting in clinical practice.

2. Describe the advantages of modular vs. template-based structured reporting.

3. Explain the importance of common data elements in standardized reporting.

Outline: There are multiple technical, conceptual, professional, and cultural reasons preventing radiologists to make use of structured reporting in their day to day practice. From the professional or clinical point of view, the flexibility of the system to provide the most relevant items while being reasonably short is the main reason why radiologists cannot report in a structured manner even if they like. Templates are the most popular containers of the predefined elements every radiologist plans to include in his/her report. But, the templates available for structured reporting are not comprehensive enough to cover all potential pathologies. In addition, there should be a basic standard to define how everybody describes a particular situation. These standards can be defined through the common data elements concept.

\section{• 10.5812/IRANJRADIOL.99303}

\section{AI in Radiology: From Theory into Practice}

Erik Ranschaert ${ }^{1, *}$

${ }^{1}$ European Society of Medical Imaging Informatics (EuSoMII), Rotterdam, The Netherlands

${ }^{*}$ Corresponding author: European Society of Medical Imaging Informatics (EuSoMII), Rotterdam, The Netherlands. Email: erik.ranschaert@gmail.com

\footnotetext{
Abstract

Background: Radiology is at the forefront of the revolution in medical imaging, which is mainly based on the progress made in machine learning and deep learning. New tools are being developed and made commercially available for implementation in radiology practice. AI solutions can intervene in different parts of the entire radiological workflow, and thus are likely to have a significant impact on the way that radiology services are being offered.

Objectives: By listening to this lecture, the audience is expected to:
}

1. Understand the basic principles of machine learning and deep learning.

2. Understand the different ways and possibilities by which these techniques can be applied in radiology.

3. Understand the advantages, disadvantages, and risks of implementing AI-based tools in radiology practice.

Outline: In this presentation, a brief historical overview is provided of the progress that has been made in the past few years in the field of artificial intelligence. The basic principles of machine learning and deep learning are explained. Radiology is at the forefront of these developments, with the ability to provide a huge resource of data. The way these new AI-based applications can be applied is explained, accompanying with advantages, disadvantages, and risks. Advice is provided on how to use these tools in clinical practice.

\section{-10.5812/IRANJRADIOL.99304 \\ AI Startups: The Need for Collaborative Research and Development}

Maedeh Sadat Tahaei ${ }^{1, *}$

${ }^{1}$ ICT Innovation Center(ICTIC), SharifUniversity of Technology, Tehran, Iran

${ }^{*}$ Corresponding author: ICT Innovation Center (ICTIC), Sharif University of Technology, Tehran, Iran.Email: ms.tahaei@ictic.sharif.edu

\section{Abstract}

Background: In recent years, the rapid development of Artificial Intelligence (AI) has had a remarkable impact on the medical imaging domain. However, there remain challenges in utilizing state-of-the-art models in clinical practice. This talk focuses on challenges faced by AI startups in using machine learning in clinical practice.

Objectives:

1. How the machine learning methods solve a real clinical problem and what are its opportunities and challenges?

2. What are the future directions of AI in medical imaging?

Outline: The first part of the talk provides an overall review of some machine learning models developed for solving medical imaging problems. The second part of the talk presents some of the main challenges in utilizing state-of-the-art machine learning in medical imaging applications. These challenges include interpreting complex models, incorporating causality in our models, working with longitudinal data, model 


\section{INVITED ABSTRACTS}

generalization, and robustness. The last part of the talk focuses on opportunities and future directions of AI in medical domain advances such as automatically identifying potential responders to treatment, leading to the possibility of personalized medicine.

\section{• 10.5812/IRANJRADIOL.99305}

\section{Medical Image Sharing with Portable Devices}

Davood Akbarnia ${ }^{1, *}$

${ }^{1}$ University of Science and Culture, Hamedan, Iran

${ }^{*}$ Corresponding author: University of Science and Culture, Hamedan, Iran.Email:biobank.nbml@gmail.com

\section{Abstract}

Background:In this presentation, we look at available solutions for medical services on portable devices and the benefits and features that are available on these devices today.

Objectives:

1. Electronic devices for medical image viewing

2. Portability advantages for image sharing

3. Image viewing features available on portable devices

Outline: Medical imaging has been evolved with the advent of electronic and digital technologies over the past decade. While films and printers were used to transmit images in the past, the use of PACS systems has been spread over the past decade and medical images have been viewed and browsed in digital devices. Nowadays, digital devices are a wide range of mobile devices -Handheld- to large devices and Wide Screens -which are daily demanded by users and professions for viewing and browsing medical images. Digital access to most up-to-date versions of patient medical images, medical reports, and disease history can accelerate the development of treatment, reduce the likelihood of medical and prescription errors, and prevent additional testing. Furthermore, digital access can save time and money for both patients and healthcare centers. On the other hand, the rapid expanding of services in imaging and medical image sharing is one of the challenges of ensuring the accuracy and integration of information and work processes in the way that professions always have access to accurate online data of patients. This data integration, in particular, should be done between PACS, RIS/ HIS systems, and imaging systems to circulate data automatically as much as possible without the need for manual data entry. To achieve this critical capability, other systems have been developed in the DICOM standard to serve as interfaces between the above elements, enabling the process of automating data transfer and integration. In the remainder of this presentation, the existing solution for the realization of a digital hospital and its capability in diagnosing and working with portable devices is outlined.

\section{-10.5812/IRANJRADIOL.99306}

\section{Domain-Specific AI Application in Medical Imaging: Use Cases}

Erik Ranschaert ${ }^{1, *}$

${ }^{1}$ European Society of Medical Imaging Informatics (EuSoMII), Rotterdam, The Netherlands

*Corresponding author: European Society of Medical Imaging Informatics (EuSoMII), Rotterdam, The Netherlands. Email: erik.ranschaert@gmail.com

Background: In the context of ongoing digitization in healthcare, due to the uprise of machine learning and deep learning, new tools are being developed for implementation in radiology practice. These AI-based applications can be used not only for image analysis in different domains, but also for other parts of the radiological workflow. This will be illustrated with several use cases.

Objectives: By listening to this lecture, the audience is expected to:

1. Understand the basic principles of machine learning and deep learning.

2. Understand the possibilities by which these techniques can intervene in different parts of the radiological workflow.

3. Understand the pathways that need to be followed for developing and implementing AIbased solutions for clinical use.

- Outline: AI-based applications can be used for many different purposes in radiology. In each clinical practice, it is essential, however, to define the right use cases for implementing such tools. Furthermore, it is crucial to evaluate the accuracy and value of these tools since the real-world data can be different from the data by which the algorithms are trained. In the Netherlands cancer institute, AI tools are being developed and tested, for both improving patient care and optimizing the radiological workflow. A concise overview is given of the potency of these new tools and different challenges that this project is being confronted with. 